\title{
Single-Particle Spectra, Pairing Correlations and Spectroscopic Properties of Actinide and Heavier Nuclei
}

\author{
P. Quentin ${ }^{1}$, L. Bonneau ${ }^{1}$, N. Minkov ${ }^{2}$, D. Ivanova ${ }^{3}$, J. Bartel $^{4}$, \\ H. Molique ${ }^{4}$, Meng-Hock Koh ${ }^{5}$
}

${ }^{1}$ CENBG, UMR5797, Université de Bordeaux, CNRS, 33170 Gradignan, France

${ }^{2}$ Institute of Nuclear Research and Nuclear Energy,

Bulgarian Academy of Sciences, Tzarigrad Road 72, BG-1784 Sofia, Bulgaria

${ }^{3}$ Military Medical Academy, Sofia, Bulgaria and

University Hospital "Saint Ekaterina", Sofia, Bulgaria

${ }^{4}$ IPHC, UMR7178, Université de Strasbourg, CNRS, 67200 Strasbourg, France

${ }^{5}$ Department of Physics, Faculty of Science, Universiti Teknologi Malaysia, 81310 Johor Bahru, Malaysia

Received 25 October 2021

doi: https://doi.org/10.55318/bgjp.2021.48.5-6.634

\begin{abstract}
We discuss the relevance of the single-particle (sp) properties of the well studied Skyrme SIII interaction to describe some spectroscopic properties of heavy nuclei within self-consistent Hartree-Fock plus BCS calculations (with blocking when needed), using a simple seniority pairing residual interaction $\left(V_{\text {res }}\right)$. To fit $V_{\text {res }}$ we took stock of the excellent consistency found in the rare-earth region between its adjustment to both moments of inertia (MoI) of well-deformed nuclei and oddeven mass differences. For simplicity reasons we thus made a fit on MoI. Our sample of nuclei was defined by all even-even isotopes of actinide and heavier elements, with two criteria: i) be well and rigidly deformed in their ground states, as assessed by a ratio $E\left(4_{1}^{+}\right) / E\left(2_{1}^{+}\right)>3.2$, ii) present some isomeric activity with suggested isomeric energy $\left(E_{\mathrm{IS}}\right)$ and spin/parity characterization. We have thus considered 14 actinide isotopes (from Uranium to Nobelium) plus ${ }^{256} \mathrm{Rf}$. With a simple law of variation of the pairing matrix elements according to $N$ or $Z$, we get a fair reproduction of MoI except around the neutron number $N=152$ where SIII exaggerates its well documented magicity (for normally deformed solutions). Such a study of MoI assesses thus also the sp spectra, of which a further evaluation of the quality is provided by the comparison of the corresponding experimental and theoretical $E_{\text {IS. }}$. Our calculations were limited to quasi-particle (uncoupled) configuration of the seniority 2 type. Preliminary results on some isomeric states of the considered isotopes will be discussed particularly by comparing them with those obtained in a particle number conserving approach.
\end{abstract}

KEY WORDS: Actinide nuclei, single-particle spectra, pairing correlations. 
Single-particle spectra, pairing correlations ...

\section{Introduction}

As a consequence of the global qualitative relevance of the mean-field approximation to account for low-energy nuclear dynamics, many spectroscopic properties are governed by single-particle (sp) properties, particularly (yet not only) by sp energy spectra. Such sp spectra are influential in two ways.

First, they are influential globally, through some averaged level density $\tilde{g}_{q}\left(e_{F}\right)$ around the Fermi energy $e_{F}$ (where the subscript $q$ refers to a given charge state, $n$ or $p$ ) which defines the intensity of pairing correlations. As well known, the average pairing matrix element $\mathcal{V}_{q}$ is roughly proportional, in the so-called uniform gap method [1], to the inverse of $\tilde{g}_{q}$ as $\mathcal{V}_{q}=1 /\left[\tilde{g}_{q}\left(e_{F}\right) \operatorname{arcsinh}\left(\Omega / \tilde{\Delta}_{q}\right)\right]$, where $\Omega$ is half the width of the interval of sp energies around the Fermi energy within which the gap equations are solved and $\tilde{\Delta}_{q}$ are the average gaps for the two charge states.

Second, sp spectra are influential locally: through the specific quantum numbers of each state, they shape many features of the nuclear spectra. One may single out the spin and parity of the ground states and the band-head spectroscopy of well-deformed odd and odd-odd nuclei, as well as those of quasi-particle (qp) isomeric states in well deformed nuclei.

In this paper, we aim at assessing the quality of sp spectra as obtained in a standard Hartree-Fock plus BCS (HF + BCS) approach, with a self-consistent blocking treatment when necessary, by checking their results with relevant data.

\section{Pairing Correlations Properties}

The pairing correlations properties accessible with such a microscopic approach are mainly (i) moments of inertia (MoI) of well rigidly deformed even-even nuclei and (ii) odd-even mass differences (OEMD) through, e.g., the three points differences

$$
\begin{aligned}
& \Delta_{n}(N, Z)=\frac{(-1)^{N}}{2}\left[S_{n}(N, Z)-S_{n}(N+1, Z)\right] \\
& \Delta_{p}(N, Z)=\frac{(-1)^{Z}}{2}\left[S_{n}(N, Z)-S_{n}(N, Z+1)\right]
\end{aligned}
$$

with odd $N$ or $Z$ respectively and where $S_{n}(N, Z)$ and $S_{p}(N, Z)$ are the neutron and proton separation energies of the nucleus defined by the nucleon numbers $N$ and $Z$.

As well known, pairing correlations generate a quenching of the total MoI. This is a consequence of the well established Coriolis Anti Pairing (CAP) collective mode of Mottelson and Valatin [2]. As explicitly demonstrated in Ref. [3] (see e.g. Figure 3 therein), one observes in rotating nuclei an intrinsic collective motion counter-rotating with respect to the global rotation, corresponding to a small 


\section{P. Quentin et al.}

"Landau" orbital diamagnetism supplemented by a CAP effect varying with the laboratory angular velocity $\Omega$ of the intrinsic frame.

Both effects on MoI and OEMD have been quoted in the seminal paper of Bohr, Mottelson and Pines [4] among the clues for the existence of pairing correlations of the BCS type in atomic nuclei. Being both rather simply accessible for a mean-field (plus correlations) approach, their reproduction allows to conclude on the quantitative validity of such a description of the pairing correlations.

Beyond these two tests, one must mention two others. The first one is the height $B$ of the inner fission barriers. Inner barriers are preferred for that purpose to outer barriers, being free of the subtleties of the different fission paths that appear at very large deformations. As a general rule, pairing acts in opposite direction to shell effects. It does thus contribute negatively to the total energy at the top of this barrier. Therefore, a good estimate of pairing correlations plays an important role to obtain correct values of these barrier heights. The second test is provided by the excitation energies $E_{\mathrm{IS}}$ of high- $K$ isomeric states. These isomeric energies may be decomposed at least qualitatively into two contributions:

$$
E_{\mathrm{IS}}=E_{\mathrm{ph}}+E_{\text {pair }}
$$

where $E_{\mathrm{ph}}$ is the increase in energy, with respect to some particle-hole qp vacuum, due to the promotion of nucleons from hole into particle states, and $E_{\text {pair }}$ is the increase in energy resulting from the lack of pairing correlations in the isomeric state with respect to the correlated ground state. The $E_{\text {pair }}$ contribution, called the Pauli blocking effect has been advocated long ago, in Refs. [5,6]. It is due to the fact that in order to generate the qp isomeric states one occupies some sp states by 1 nucleon, thus preventing the promotion of pairs into these states.

To be complete, one must remark that what is called a pair here does not correspond to a set of two time-reversal conjugate sp states, since this partial occupation of two sp levels breaks the Kramers degeneracy. It appears, however, that this symmetry violation is weak enough so that one can always identify unambiguously quasi-pairs (see e.g. Ref. [7]).

One may illustrate the above for instance in the case of the $16^{+}$isomeric state of ${ }^{178} \mathrm{Hf}$. In the calculations of Ref. [8] performed within a particle-number conserving Highly Truncated Diagonalization Approach (HTDA) formalism (see below), the two components of $E_{\mathrm{IS}}$ are additive and one finds $E_{\mathrm{IS}}^{(\text {th })}=2.6 \mathrm{MeV}$ (as compared to $E_{\mathrm{IS}}^{(\exp )}=2.45 \mathrm{MeV}$ ), which is shared between $E_{p h}=1.5 \mathrm{MeV}$ and $E_{\text {pair }}=1.1 \mathrm{MeV}$.

It is then clear that the intensity of pairing correlations in both the ground and isomeric states plays a role in determining the value of $E_{\mathrm{IS}}^{(\mathrm{th})}$. 
Single-particle spectra, pairing correlations ...

\section{Single-Particle Spectral Properties}

The single-particle spectra determine the ground-state quantum numbers $I$ and $\pi$ of odd nuclei, and less directly those of odd-odd nuclei. A systematic assessment of the relevance in this respect of some Skyrme force parametrizations has been performed in Ref. [9] for the 621 odd $-N$ and odd- $Z$ nuclei quoted in the NUBASE2003 [10]. A pairing treatment using a $\left|T_{z}\right|=1$ schematic seniority force was included. Apart from spherical nuclei, axial and intrinsic parity symmetry were imposed on calculated non-spherical nuclei. Among these deformed nuclei only those for which the prolate-oblate energy difference was larger than 2 $\mathrm{MeV}$ and the absolute value of the usual quadrupole parameter $\beta$ larger than 0.1 , were retained to eliminate soft nuclei and those presenting potentially a shape coexistence. The spin $I$ and parity $\pi$ of spherical nuclei were determined by the total angular momentum $j$ and the parity of the orbit of the unpaired nucleon. Thus, one assumes there $I=j$ and $\pi=(-1)^{l}$ where $l$ is the orbital angular momentum quantum number of the considered orbit. For deformed nuclei, we consider the lowest 1 qp configuration obtained as a self-consistent solution of the odd system within the equal filling approximation as detailed, e.g., in Ref. [11]. There, the ground-state quantum numbers were given by the conserved projection $K$ of the angular momentum on the symmetry axis (thus $I=K$, possible exception for $K=1 / 2$ ) and the intrinsic parity $\pi$ of the retained qp state. As a result we have been able to reproduce the exact quantum numbers for about $2 / 3$ of the selected nuclei for the SIII interaction [12] and slightly less for the $\mathrm{SkM}^{*}$ interaction [13]. This compares favorably (see Ref. [9]) with what has been achieved within a more phenomenological approach of the macroscopicmicroscopic type, using the Finite Range Droplet Model [14] supplemented by shell-energy corrections obtained from folded Yukawa mean fields [15].

The sp spectra determine also to a large extent the band-head spectroscopy of deformed odd nuclei. The quality of their description within the Bohr-Mottelson unified model upon using seniority-1 qp-state wavefunctions to describe the intrinsic degrees of freedom, has been assessed in Ref. [16]. In these selfconsistent $\mathrm{HF}+\mathrm{BCS}$ blocking calculations, the Skyrme SIII interaction has been used with a schematic $\left|T_{z}\right|=1$ seniority force. For a limited sample of deformed odd- $N$ and odd- $Z$ nuclei in the actinide region, it has been found that in the case of neutron $q p$ excited states the relative energies are reproduced within a couple of $100 \mathrm{keV}$ or less (limiting oneself to excitation energies of the order of the pairing gap in view of the limitation to seniority-1 excitations with respect to the even-even core). The agreement for proton qp excited states has been found to be less favourable. This may be related to the fact that we have used the Slater approximation to evaluate the exchange contribution of the Coulomb 2-body interaction to the proton mean field (yielding a spurious systematic underestimation of all particle-hole energies) [17]. Similar calculations performed with the Skyrme $\mathrm{SkM}^{*}$ interaction [13] are systematically yielding a slightly inferior agreement. 


\section{P. Quentin et al.}

One may ask whether the above conclusions, based on normally-deformed solutions, would remain valid when reaching the super-deformed region. Calculations performed with three Skyrme force parametrizations SIII, SkM* and SLy5* [18] have actually yielded similar conclusions (see Refs. [19,20] for such spectroscopic properties in the second well of odd fissioning systems).

Finally the sp spectra govern the occurrence, energies and features of qp isomeric states known as high- $K$ isomers in (axially) deformed nuclei as it will be illustrated below in detail.

\section{Choice of the Particle-Hole Interaction}

In order to make a relevant assessment of the impact of sp spectra on specific spectroscopic properties, one should first make use of a phenomenological effective particle-hole interaction for which an extended global study of these properties is available. This is the case of the old (first used in 1973) SIII Skyrme parametrization [21]. It clearly has well-known deficiencies, as too high nuclearmatter incompressibility modulus $(K=355.4 \mathrm{MeV})$ noting that it was fitted before data on isoscalar monopole giant resonance were available. It also yields too large charge radii in medium and heavy nuclei (e.g. $5.57 \mathrm{fm}$ for ${ }^{208} \mathrm{~Pb}$ vs $5.50 \mathrm{fm}$ experimentally) and exaggerates the heights of the first fission barrier in actinide nuclei. For instance, a defect of about $15 \%$ of the fissility parameter in ${ }^{240} \mathrm{Pu}$ was estimated in Ref. [13]. Yet, it provides an excellent reproduction of the ground-state deformation properties of even-even nuclei (see, e.g., [21]), a reasonable account of magnetic moments of deformed odd nuclei (see, e.g., [7]) and, as we have seen above, many other spectroscopic properties.

It is interesting to compare in that respect, the SIII and SkM* parametrizations. The latter has indeed corrected the three previously quoted deficiencies of the bulk properties of the former (compressibility modulus of $216.7 \mathrm{MeV}$ instead of $355.4 \mathrm{MeV}$, some measure of the surface-energy parameter of $17.51 \mathrm{MeV}$ instead of $18.33 \mathrm{MeV}$ and a charge radius in ${ }^{208} \mathrm{~Pb}$ of $5.49 \mathrm{fm}$ instead of $5.57 \mathrm{fm}$ ). However as discussed in the previous section it yields generally a slightly less good reproduction of some spectroscopic properties. Making merely a fit on some bulk properties is clearly by far not enough to warrant its spectroscopic relevance.

\section{Choice of the Retained Sample of Nuclei}

We have performed the present study in a sample comprising at least all actinide and heavier isotopes satisfying the two following conditions:

- being well and rigidly deformed with a ratio $R$ of the energies of their first $4^{+}$and $2^{+}$states such that $R \geq 3.2$,

- known to have an isomeric activity with a well characterized or strongly 
Single-particle spectra, pairing correlations ...

suggested spin and parity whose isomeric energy $E_{\mathrm{IS}}$ is reported in the 2015 evaluation of Ref. [22].

To those we add one isotope $\left({ }^{238} \mathrm{Pu}\right)$ to complete an isotopic $(\mathrm{Z}=94)$ series and another $\left({ }^{244} \mathrm{Pu}\right)$ Ref. [23] to complete an isotonic series $(N=150)$. This leaves us with fourteen nuclei: ${ }^{234,236} \mathrm{U},{ }^{236,238,240,244} \mathrm{Pu},{ }^{244,246} \mathrm{Cm},{ }^{248} \mathrm{Cf}$, ${ }^{250,256} \mathrm{Fm},{ }^{252,254}$ No and ${ }^{256} \mathrm{Rf}$.

\section{Choice of the Particle-Particle, Hole-Hole Interaction}

We use a schematic "seniority" force, assuming constant pairing matrix elements between sp eigenstates $i, j$ of the 1-body density matrix (defining the canonical basis) of a charge state $q$, restricted to those whose sp energy $e$ is such that $e \leq$ $\lambda_{q}+6 \mathrm{MeV}$ where $\lambda_{q}$ is the corresponding chemical potential. We parametrize it as [24]

$$
\forall i, j, \quad\left\langle i \bar{i}\left|V_{\text {res }}^{(q)}\right| j \bar{j}\right\rangle-\left\langle i \bar{i}\left|V_{\text {res }}^{(q)}\right| \bar{j} j\right\rangle=\frac{G_{q}}{N_{q}+11},
$$

where $N_{q}$ is the number of nucleons of charge state $q$ in the considered nucleus. It has recently been shown [25] that fitting $G_{q}$ on either MoI or OEMD for well and rigidly deformed rare-earth even-even isotopes yields reasonably consistent HF + BCS results. Consequently in view of the easier MoI fit (having to perform only calculations for even-even isotopes) as opposed to the OEMD fit (necessitating at least supplementary calculations of two odd-even nuclei adjacent to each considered even-even nucleus) we have adjusted for the 14 actinide and heavier isotopes presented in the previous section, the seniority force parameters to reproduce roughly the data on $\mathrm{MoI}$ as deduced from the simple rotor expression for the energy of the first $2^{+}$state $E_{2}=6 \hbar^{2} / 2 \mathcal{J}_{2}$, where $\mathcal{J}_{2}$ is the MoI associated with this $2^{+}$state.

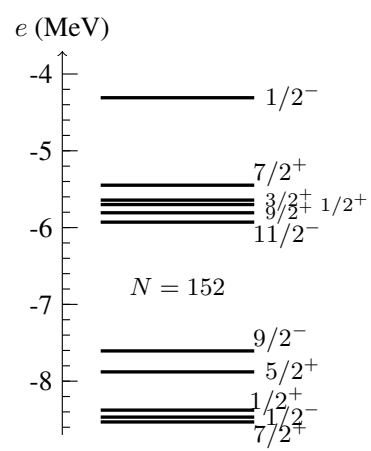

Figure 1. Neutron single-particle energy spectrum around $N=152$ from the groundstate solution of ${ }^{254} \mathrm{No}$. 


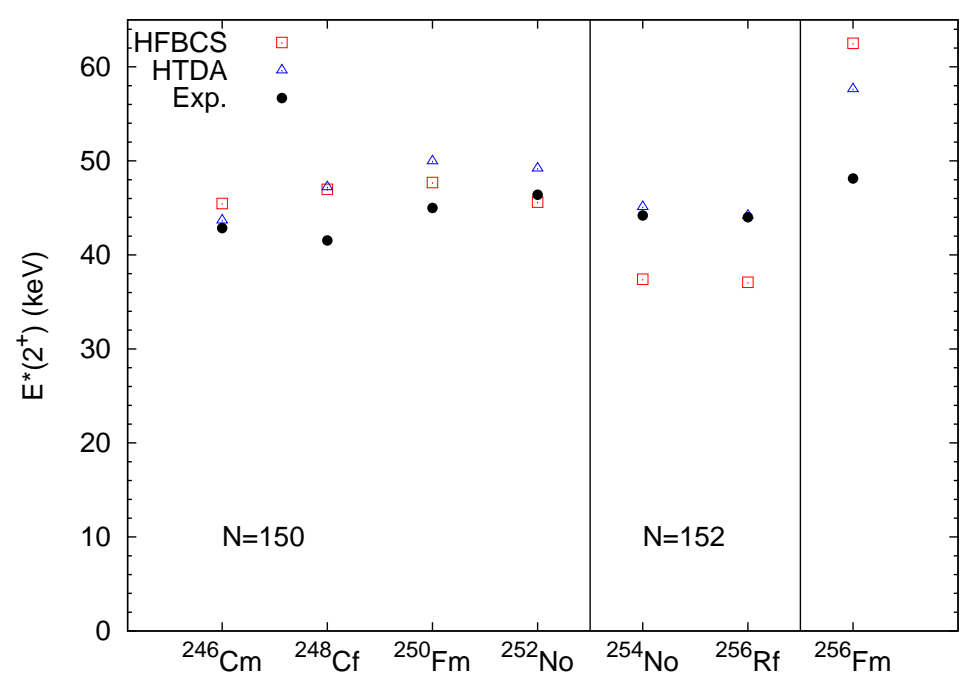

Figure 2. (Color online) Excitation energies of the first $2^{+}$state, in a sample of nuclei, calculated within the HFBCS and HTDA approaches, compared with experimental values.

These MoI are calculated à la Inglis-Belyaev [26] adding to the results a phenomenological Thouless-Valatin correction (see e.g. [27]) in the form of a multiplying factor of 1.32 as proposed in Ref. [28]. We chose $G_{n}=-15.8 \mathrm{MeV}$ for neutrons and $G_{p}=0.9 \times G_{n}=-14.22 \mathrm{MeV}$ for protons.

The agreement with the data on MoI is consistently rather good below the $N=$ 152 deformed magic gap. The rms deviation from data of the energy of the first $2^{+}$state is found to be $2.67 \mathrm{keV}$, corresponding to a relative error of about $6 \%$. For $N=152$ it appears that the sp neutron energy gap is significantly too large (see Figure 1). This entails a deficit in the pairing gap for $N=152$ nuclei (see Figure 2). Correlatively neutron levels above $N=152$ are pushed upwards yielding too large pairing correlations there and thus too small MoI values, resulting in too large $2^{+}$energies as also seen on Figure 2.

\section{Isomeric Energies}

Even though, as we just discussed above, the behaviour of MoI constitutes a good indicator of the validity of our sp spectra, a tougher and more selective test is provided by the isomeric energies of high- $K$ seniority 2 isomers. It is tougher (or more detailed) because it involves merely a couple of sp energies whereas MoI is mostly concerned with a bunch of sp states near the Fermi level. It is also more selective (charge-wise) in that it provides separate tests on proton and neutrons, as opposed to the MoI where one is summing both charge contributions. 
Single-particle spectra, pairing correlations ...

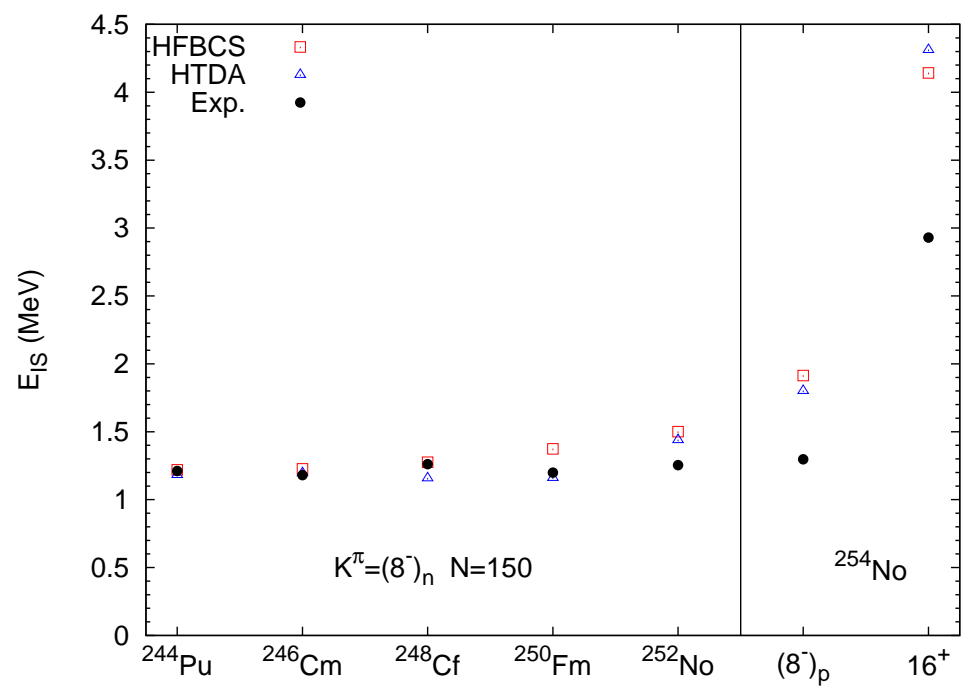

Figure 3. (Color online) Isomeric energies in a sample of nuclei, calculated within the HFBCS and HTDA approaches, compared with experimental values.

As seen in Figure 3, we have obtained within our $\mathrm{HF}+\mathrm{BCS}$ calculations a rather satisfactory description of the isotonic $(N=150)$ series of neutron $\left(9 / 2^{-}, 7 / 2^{+}\right) 8^{-}$isomeric states from ${ }^{244} \mathrm{Pu}$ up to ${ }^{252} \mathrm{No}$, even though it consistently deteriorates upon approaching $Z=102$.

In ${ }^{254} \mathrm{No}$ we found three $8^{-}$qp configurations: the lowest one corresponding to a proton $\left(9 / 2^{+}, 7 / 2^{-}\right)$excitation, a $\left(11 / 2^{-}, 5 / 2^{+}\right)$neutron state lying 440 $\mathrm{keV}$ above the proton state and another neutron excitation $\left(11 / 2^{-}, 5 / 2^{+}\right)$still $210 \mathrm{keV}$ higher. The excitation energy of the proton configurations is found much too high (by about $600 \mathrm{keV}$ ) with respect to the experimental isomeric energy. A coupling of this state with the neutron states, pushing it down, is plausible, yet unable to explain the discrepancy. Interestingly, it has been claimed in Ref. [29] that an isomeric activity observed at about twice the energy of the $8^{-}$ proton qp state could correspond to a $16^{+}$state. Similarly to what is found in the ${ }^{178} \mathrm{Hf}$ case such an isomeric state would be built as the combination of a neutron and a proton $8^{-}$states (see, e.g., Ref. [8] for a discussion of the ${ }^{178} \mathrm{Hf}$ case). We obtain in our calculations such a state yet much higher in excitation energy than what is assumed, due to both the too high proton $8^{-}$isomeric energy (as just above discussed) and a similar defect for the neutron state due to the too large gap above the last occupied state for 152 neutrons. 


\section{Preliminary Results of a Particle Number Conserving Approach}

As already discussed, the Pauli blocking effect quenches pairing correlations for the nucleons of the charge states within which a seniority-2 excitation is performed. As well known (see, e.g., [30]) the BCS approximation spuriously enhances the decrease of pairing correlations in such a low-correlation regime. Actually in most of our self-consistent BCS solutions the superfluid character is lost for the blocked-nucleon charge state in the qp excited configurations. The question therefore arises of assessing quantitatively how much this would affect the determination of the isomeric states. To answer that question, we have performed calculations within an approach explicitly conserving the particle number, dubbed as the Highly Truncated Diagonalization Approach (HTDA) which makes use of the same particle-hole interaction as the one used in the HF + BCS case. As for the particle-particle hole-hole interaction $V_{\text {res }}$, we have taken a zerorange (charge-independent) $\left|T_{z}\right|=1$ interaction whose intensity is adjusted to obtain similar quality in the reproduction of $\mathrm{MoI}$ as before.

It is beyond the scope of this paper to review in detail the HTDA formalism. This is to be found, for instance, in the subsection II A of Ref. [31]. It suffices here to recall that from a Slater determinant vacuum defined from the above discussed good quality HF + BCS mean fields, we treat the pairing correlations by allowing all 1 and 2 "pair" transfers in a valence space of canonical basis states with a Hamiltonian composed of an independent qp (of particle-hole type) part and the zero range $V_{\text {res }}$ interaction. Actually due to our treatment of the core polarization induced by the self-consistent treatment of the qp excitations, the Cooper pairs are composed of sp states which are nearly time-reversal conjugated, as already mentioned. The moments of inertia are evaluated (within the Inglis-Belyaev plus Thouless-Valatin approach as before) using eigenstates of the resulting one-body density matrix to define the occupation probabilities and computing the BCS gaps from matrix elements of the $V_{\text {res }}$ interaction between canonical basis sp states.

As illustrated in Figure 2, we obtain the same kind of agreement with data on the MoI as achieved within the HF + BCS approach, except for a slight improvement near the $N=152$ deformed magic gap. A similar conclusion may be drawn as far as the isomeric energies are concerned (see Figure 3), noting merely a very small decrease of these energies with respect to the HF + BCS approach. This very limited effect of the HTDA reduction of the pairing gap might be qualitatively explained in terms of a qp energy approximation of the isomeric energy. Indeed in so far as the energy difference between the Fermi energy and the relevant sp energies would be significantly larger than the quenched (yet non vanishing) pairing gap, both quantities being involved through their square, the pairing-gap term would be dominated by the sp-energy term. 
Single-particle spectra, pairing correlations ...

\section{Conclusions}

Some conclusions may be drawn from the present study. Moments of inertia of well-deformed actinide nuclei have been generally well reproduced within a $\mathrm{HF}+\mathrm{BCS}$ approach using a well-established Skyrme force parametrization and a schematic pairing residual interaction of the seniority type.

Taking stock of this, whenever this agreement is of a lesser quality, one may infer that the sp spectra are not adequately represented. This has been shown (using the SIII parametrization) to be the case in the vicinity of the $N=152$ deformed magic number.

A more detailed and thus stringent test of sp spectra is provided by the consideration of the isomeric energies of seniority- 2 high- $K$ isomers. The conclusions drawn from the comparison of MoI with data are reinforced by such a comparison. Whenever the MoI are well reproduced, the agreement for these excitation energies is quanlitatively very good.

Finally we may deliver a caveat emptor as far as the fit of particle-hole interactions is concerned. In so far as one is interested in the spectroscopic properties associated with a given phenomenological effective force, one should not satisfy oneself with merely searching for an adjustment of some bulk nuclear properties. Finer details of sp spectra escape from such a global approach. Whereas welldeformed nuclei constitute the best laboratory for studying these spectroscopic properties within a mean-field-plus-pairing-correlations approach (minimizing the effect of quantal fluctuations of the nuclear shape), it is impossible to avoid their specific and as systematic as possible studies.

\section{Acknowledgements}

This work has been done in the framework of and supported by the PHC Rila 2019 France-Bulgaria agreement under contract number 43184NJ and contract No. KP-06-RILA/6 with the Bulgarian National Science Fund (BNSF) which are gratefully acknowledged. One author (M.H.K.) would like to acknowledge the Ministry of Education Malaysia for the financial support through the Fundamental Research Grant Scheme (FRGS/1/2018/ST/G02/UTM/02/6) and UTM (R.J130000.7854.5F028).

\section{References}

[1] M. Brack et al. (1972) Rev. Mod. Phys. 44320 (1972).

[2] Å. Bohr, B.R. Mottelson and D. Pines (1958) Phys. Rev. 110936.

[3] H. Laftchiev, D. Samsoen, P. Quentin and I.N. Mikhailov (2003) Phys. Rev. C 67 014307.

[4] B.R. Mottelson, and A.C. Valatin (1960) Phys. Rev. Lett. 5511.

[5] V.G. Soloviev (1961) Kgl. Dan. Vid. Selsk. Mat. Fys. Skr. 1 (11) 1. 


\section{P. Quentin et al.}

[6] S.G. Nilsson and O. Prior (1961) Mat. Fys. Medd. Dan. Vid. Selsk. 32(16) 1.

[7] L. Bonneau, N. Minkov, Dao Duy Duc, P. Quentin and J. Bartel (2015) Phys. Rev. C 91054307.

[8] N. Pillet, P. Quentin and J. Libert (2002) Nucl. Phys. A 697141.

[9] L. Bonneau, P. Quentin and P. Möller (2007) Phys. Rev. C 76024320.

[10] G. Audi, O. Bersillon, J. Blachot and A.H. Wapstra (2003) Nucl. Phys. A 729141.

[11] S. Perez-Martin and L.M. Robledo (2008) Phys. Rev. C 78014304.

[12] M. Beiner, H. Flocard, Nguyen Van Giai and P. Quentin (1975) Nucl. Phys. A 238 29.

[13] J. Bartel, P. Quentin, M. Brack, C. Guet and H.-B. Håkansson (1982) Nucl. Phys. A 28679.

[14] J.R. Nix and P. Möller (1990) Nucl. Phys. A 520 c369.

[15] M. Bolsterli, E.O. Fiset, J.R. Nix and J.L. Norton (1972) Phys. Rev. C 51050.

[16] Meng-Hock Koh, Dao Duy Duc, T.V. Nhan Hao, Ha Thuy Long, P. Quentin and L. Bonneau (2016) Eur. Phys. J. A 525.

[17] J. Le Bloas, Meng-Hock Koh, P. Quentin, L. Bonneau and J.I. Ithnin (2015) Phys. Rev. C 91054307.

[18] A. Pastore, D. Davesne, K. Bennaceur, J. Meyer and V. Hellemans (2013) Phys. Scr. $T 154014014$

[19] J. Libert, M. Meyer and P. Quentin (2017) Phys. Rev. C 76024320.

[20] Meng-Hock Koh, L. Bonneau, P. Quentin, T.V. Nhan Hao and Husin Wagiran (2017) Phys. Rev. C 95014315

[21] H. Flocard, P. Quentin and D. Vautherin (1973) Phys. Lett. B 46303.

[22] A.K. Jain, B. Maheshwari, S. Garg, M. Patial and B. Singh (2015) Nucl. Data Sheets 1281.

[23] S. Hota, et al. (2016) Phys. Rev. C 94 081303(R).

[24] P. Bonche, H. Flocard, P.-H. Heenen, S.J. Krieger and M.S. Weiss (1985) Nucl. Phys. A 44339.

[25] Nurhafiza M. Nor, Nor-Anita Rezle, Kai-Wen Kelvin-Lee, Meng-Hock Koh, L. Bonneau and P. Quentin (2019) Phys. Rev. C 99064306.

[26] S.T. Belyaev (1961) Nucl. Phys. 24322.

[27] E.K. Yuldashbaeva, J. Libert, P. Quentin and M. Girod (1999) Phys. Lett. B 4611.

[28] J. Libert, M. Girod and J.P. Delaroche (1999) Phys. Rev. C 60054301.

[29] R.M. Clark et al. (2010) Phys. Lett. B 69019.

[30] D.C. Zheng D.W.L. Sprung and H. Flocard (1992) Phys. Rev. C 461355.

[31] J. Le Bloas, L. Bonneau, P. Quentin, J. Bartel and D.D. Strottman (2012) Phys. Rev. C 86034332. 